\title{
Fully automated quantification of left ventricular volumes and function in cardiac MRI: clinical evaluation of a deep learning-based algorithm
}

\author{
Benjamin Böttcher ${ }^{1} \cdot$ Ebba Beller $^{1} \cdot$ Anke Busse $^{1} \cdot$ Daniel Cantré $^{1} \cdot$ Seyrani Yücel ${ }^{2} \cdot$ Alper Öner $^{2} \cdot$ Hüseyin Ince $^{2}$. \\ Marc-André Weber ${ }^{1}$ (i) $\cdot$ Felix G. Meinel ${ }^{1}$ (1)
}

Received: 15 April 2020 / Accepted: 6 July 2020 / Published online: 16 July 2020

(c) The Author(s) 2020

\begin{abstract}
To investigate the performance of a deep learning-based algorithm for fully automated quantification of left ventricular (LV) volumes and function in cardiac MRI. We retrospectively analysed MR examinations of 50 patients (74\% men, median age 57 years). The most common indications were known or suspected ischemic heart disease, cardiomyopathies or myocarditis. Fully automated analysis of LV volumes and function was performed using a deep learning-based algorithm. The analysis was subsequently corrected by a senior cardiovascular radiologist. Manual volumetric analysis was performed by two radiology trainees. Volumetric results were compared using Bland-Altman statistics and intra-class correlation coefficient. The frequency of clinically relevant differences was analysed using re-classification rates. The fully automated volumetric analysis was completed in a median of $8 \mathrm{~s}$. With expert review and corrections, the analysis required a median of $110 \mathrm{~s}$. Median time required for manual analysis was $3.5 \mathrm{~min}$ for a cardiovascular imaging fellow and $9 \mathrm{~min}$ for a radiology resident $(\mathrm{p}<0.0001$ for all comparisons). The correlation between fully automated results and expert-corrected results was very strong with intra-class correlation coefficients of 0.998 for end-diastolic volume, 0.997 for end-systolic volume, 0.899 for stroke volume, 0.972 for ejection fraction and 0.991 for myocardial mass (all $\mathrm{p}<0.001$ ). Clinically meaningful differences between fully automated and expert corrected results occurred in $18 \%$ of cases, comparable to the rate between the two manual readers (20\%). Deep learning-based fully automated analysis of LV volumes and function is feasible, time-efficient and highly accurate. Clinically relevant corrections are required in a minority of cases.
\end{abstract}

Keywords Cardiac magnetic resonance imaging $\cdot$ Quantitative analysis $\cdot$ Left ventricle $\cdot$ Deep learning

Electronic supplementary material The online version of this article (https://doi.org/10.1007/s10554-020-01935-0) contains supplementary material, which is available to authorized users.

Felix G. Meinel

felix.meinel@med.uni-rostock.de

1 Institute of Diagnostic and Interventional Radiology, Paediatric Radiology and Neuroradiology, University Medical Centre Rostock, Ernst-Heydemann-Str. 6, 18057 Rostock, Germany

2 Department of Internal Medicine, Divison of Cardiology, University Medical Center Rostock, Rostock, Germany

\section{Introduction}

The quantification of $\mathrm{LV}$ ejection fraction (EF) as a measure of global systolic LV function is clinically important in a wide spectrum of cardiac conditions. LV EF is a strong prognostic parameter in acute coronary syndrome $[1,2]$, acute myocarditis [3] and heart failure [4]. In heart failure, EF serves to distinguish heart failure with reduced, mid-range or preserved $\mathrm{EF}$ - which are considered separate conditions with specific treatment recommendations according to the most recent European Society of Cardiology guidelines [5]. Precise quantification of EF is also important for the indication of implantable cardioverter-defibrillators or cardiac resynchronization therapy in both ischemic and nonischemic heart disease [6].

Cardiac magnetic resonance (CMR) imaging is established as the clinical gold standard for the evaluation of left 
ventricular (LV) volumes, ejection fraction and myocardial mass $[5,7]$. In order to derive end-diastolic volume (EDV), end-systolic volume (ESV), stroke volume (SV) and ultimately EF from CMR datasets, the epicardial and endocardial contours of the myocardium need to be defined for each short-axis slice in diastole and systole. Manual contouring is time-consuming and prone to inter-observer variability. Many vendors now offer software tools for semi-automated volumetric analysis.

More recently, the dramatic evolution in artificial intelligence-specifically deep learning-has enabled the development of fully automated algorithms for volumetric analysis of CMR datasets. Initials studies indicate substantial time-saving and reduced observer bias compared to manual or semi-automated contouring [8, 9-14]. Some of these studies used pre-processed datasets, CMR examinations of healthy individuals from population-based studies or highly selected patient populations. The purpose of our investigation was to investigate the performance of a deep learning-based algorithm for fully automated quantification of left ventricular volumes and function in CMR examinations across a spectrum of cases typically encountered in clinical routine.

\section{Material and methods}

\section{Ethical approval, study design and patient selection}

The study was approved by the responsible institutional review board, informed consent was waived. The study was designed as a retrospective single centre cohort study. The study population consisted of 50 adult patients who underwent a CMR examination at $1.5 \mathrm{~T}$ in 2018 . We selected suitable patients through a retrospective search of our radiology information system. We excluded children $(n=8)$, patients examined at $3 \mathrm{~T}(\mathrm{n}=8)$, patients with severe congenital heart disease $(n=4)$, large left ventricular aneurysms $(n=2)$ and datasets with severe artefacts $(n=15)$.

\section{Cardiac MR technique}

All MRI examinations were performed on a $1.5 \mathrm{~T}$ MRI scanner (Avanto fit, Siemens Healthineers). The cardiac MRI protocol was tailored to the clinical indication following national and international recommendations. All protocols included steady-state free precession cine sequences in 4-chamber view, 2-chamber view, left ventricular outflow tract view and as short axis stack perpendicular to the long axis of the LV. For the short-axis cine images, in-plane resolution was $1.9 \times 1.9 \mathrm{~mm}$, slice thickness was $8 \mathrm{~mm}$ with an inter-slice gap of $2 \mathrm{~mm}$. TR was $42 \mathrm{~ms}$, TE $1.1 \mathrm{~ms}$ and flip angle $72^{\circ}$. Parallel acquisition (GRAPPA) was used with an acceleration factor of 2 . The short-axis image stack typically consisted of 10 slices. FOV size was typically $360 \mathrm{~mm}$. Retrospective ECG-gating was used with 25 phases calculated over the cardiac cycle.

\section{Fully automated volumetric analysis}

The fully automated volumetric analysis of LV volumes and function was performed using a novel deep learning-based algorithm within a dedicated commercially available software (cvi42, Version 5.10.1, Circle Cardiovascular Imaging Inc.). In brief, the network architecture is inspired by Unet architecture that is widely used for medical image segmentation. The architecture is adapted to maximize performance on CMR images in clinical settings (fast and low memory requirement). The network had been trained on 5000 healthy subjects from UK Biobank dataset combined with pathological cases collected from clinical collaborators. Manual annotation of the training set included the LV endocardium, LV epicardium, and RV endocardium.

The short-axis cine stack was manually selected, and the deep learning-based algorithm was started with one mouse click. No other manual pre-processing or user interaction occurred. The fully automated algorithm automatically identified the end-diastolic and end-systolic phases and performed complete contouring of the endocardial contour in both diastole and systole. The epicardial contour for calculation of LV mass was automatically delineated in diastole only. The papillary muscles were detected and not included with the LV volume. Time required for fully automated volumetric analysis was recorded.

\section{Expert-corrected automated volumetric analysis}

After recording the results and contours of the fully automated analysis, the selection of cardiac phases, selection of slice inclusion and the contours were subsequently checked and corrected by an expert (board-certified radiologist with subspecialisation in cardiovascular imaging and level III certification for cardiac MR and CT, initials blinded) as necessary. The number and type of corrections were registered categorised in correction of cardiac phase (choice of end-systolic or end-diastolic phase), correction of slice inclusion (apical/basal) and fine corrections of contours. The time required for all checks and corrections was recorded and added to the time required for fully automated analysis, to determine the time required for expert-corrected automated analysis.

\section{Manual volumetric analysis}

Manual volumetric analysis was performed on a randomly selected subset of 25 cases by a radiology resident (initials 
blinded) and a board certified radiologist currently performing a fellowship in cardiovascular radiology (initials blinded) using manual contouring tools within the same software (cvi42, Version 5.10.1, Circle Cardiovascular Imaging Inc.). The analysis was performed on the short axis cine stack. The 4-chamber cine sequence was displayed for reference with a reference line indicating the position of the short axis slice to determine the most basal and apical slices to be included into the left ventricle. First, the end-systolic and end-diastolic phases were visually determined followed by manual delineation of the epi- and endocardial contours in end-diastole and the endocardial contours in end-systole. Both readers had been instructed in the use of the software. The cardiovascular imaging fellow had significant experience in performing volumetric analysis with this software (approximately 50 cases), whereas the radiology resident had limited experience (approximately 10 cases). Readers generally used a threshold-based tool for segmentation of the endocardial border and a multi-point tool for segmentation of the epicardial border but were free to use whichever tools in the software they found most efficient and accurate. Readers were instructed to exclude papillary muscles from left ventricular volume. The time needed for manual analysis was recorded.

\section{Analysis of clinical data}

Analysis of electronic patient data was performed to assemble demographic information as well as indications and results of the CMR examinations.

\section{Statistical analysis}

Statistical analysis was performed with Prism (version 8.2.1, GraphPad Software Inc.) and SPSS Statistics (version 25, IBM). Continuous values were presented as median and interquartile range, since normal distribution could not be assumed. Categorical data were displayed as absolute frequencies and proportions. Pairwise comparison of numerical results between volumetric methods was made using Wilcoxon matched pairs test. Inter-method agreement was assessed using Bland-Altmann statistics and intra-class correlation coefficient for absolute agreement with a twoway mixed model. To account for multiple testing, P-values $<0.005$ were considered to indicate statistical significance. Intra-class correlation coefficients were considered significantly different if $95 \%$ confidence intervals did not overlap.

To evaluate the frequency of clinically important differences between fully automated and expert-corrected results, we further categorized LV ejection fraction into five categories inspired by the recommendations of the American College of Cardiology for the measure reporting in outpatient setting: hyperdynamic ( $>70 \%)$, normal (50-70\%), mild dysfunction (40-49\%), moderate dysfunction (30-39\%), severe dysfunction $(<30 \%)$ [15]. Differences leading to re-classification of a patient's LV function in a different category were defined as clinically important. We therefore analyzed the reclassification rate, i.e. how frequently expert corrections of the fully automated contours resulted in a different category of the patient's LV function, and compared this to the reclassification rate between the two manual readers.

\section{Results}

\section{Patient characteristics and spectrum of indications}

An overview of patient characteristics and CMR indications is given in Table 1. The majority of the 50 patients were male (74\%). Median age was 57 years, with a range from 18 to 80 years. Median BMI was $27.3 \mathrm{~kg} / \mathrm{m}^{2}$ with a range of 18.3 to $43.3 \mathrm{~kg} / \mathrm{m}^{2}$. The most common primary indications for CMR examinations were known or suspected ischemic heart disease $(\mathrm{n}=20,40 \%)$, known or suspected cardiomyopathy $(\mathrm{n}=18,36 \%)$ and known or suspected myocarditis
Table 1 Patient characteristics and CMR indications

\begin{tabular}{|c|c|c|c|c|c|c|}
\hline & \multicolumn{2}{|l|}{ All patients } & \multicolumn{2}{|l|}{ Women } & \multicolumn{2}{|l|}{ Men } \\
\hline & $\mathrm{n}$ & $\%$ & $\mathrm{n}$ & $\%$ & $\mathrm{n}$ & $\%$ \\
\hline Demographics & 50 & 100 & 13 & 26 & 37 & 74 \\
\hline $\begin{array}{l}\text { Age (years) } \\
\text { Median (interquartile range) }\end{array}$ & $\begin{array}{l}57 \\
(46-63)\end{array}$ & & $\begin{array}{l}48 \\
(37-58)\end{array}$ & & $\begin{array}{l}58 \\
(48-68)\end{array}$ & \\
\hline $\begin{array}{l}\text { BMI }\left(\mathrm{kg} / \mathrm{m}^{2}\right) \\
\text { Median (interquartile range) }\end{array}$ & $\begin{array}{l}27.3 \\
(25.1-30.5)\end{array}$ & & $\begin{array}{l}25.5 \\
(21.4-29.5)\end{array}$ & & $\begin{array}{l}27.5 \\
(25.5-30.6)\end{array}$ & \\
\hline \multicolumn{7}{|l|}{ Main indication for CMR } \\
\hline Known or suspected ischemic heart disease & 20 & 40 & 4 & 31 & 16 & 43 \\
\hline Known or suspected cardiomyopathy & 18 & 36 & 6 & 46 & 12 & 32 \\
\hline Known or suspected myocarditis & 11 & 22 & 3 & 23 & 8 & 22 \\
\hline Other & 1 & 2 & 0 & 0 & 1 & 3 \\
\hline
\end{tabular}


$(\mathrm{n}=11,22 \%)$. The most frequent indication in women was cardiomyopathy (6 of 13,46\%), while ischemic heart disease was the leading indication in men (16 of $37,43 \%$ ).

\section{Findings at CMR}

The results of the CMR examinations are summarized in Table 2. In 16 cases (32\%), CMR demonstrated no pathological findings. Findings consistent with ischemic heart disease were present in 16 patients (32\%). Non-ischemic cardiomyopathy was diagnosed in 11 patients $(22 \%)$, myocarditis in 3 patients $(6 \%)$ and valvular heart disease in three patients (6\%). The 12 patients with cardiomyopathies included eight patients with dilatated cardiomyopathy, one patient with hypertrophic obstructive cardiomyopathy and two patients with stress (Takotsubo) cardiomyopathy.

\section{Performance of fully automated algorithm-number and types of expert corrections}

In all cases the fully automated algorithm operated without processing failures and the LV was appropriately detected on most slices. In $20 \%$ of all data sets $(n=10)$, the expert fully agreed with the fully automated algorithm and no corrections were necessary. An example is shown in Fig. 1. In the remaining $80 \%$ of cases $(n=40)$, corrections were made on a median of 2 slices, with an interquartile range of $1-4$. Choice of end-systolic phase was corrected in 14 cases (28\%), choice of end-diastolic phase was corrected in 3 cases $(6 \%)$. Changes related to slice inclusion at the apex of the LV (adding or deleting a slice to be included) were made in 25 cases $(50 \%)$, related to slice inclusion at the base of the LV in 5 cases (10\%). Fine corrections of contours were performed in 28 cases ( $56 \%$, Fig. 2). Incorrect contouring of the RV instead of the LV on one apical slice occurred in one case (Fig. 2).

Table 2 Main findings at CMR

\begin{tabular}{lcc}
\hline & $\mathrm{n}$ & $\%$ \\
\hline No pathological findings & 16 & 32 \\
Ischemic heart disease & 16 & 32 \\
Cardiomyopathy & 11 & 22 \\
DCM & 8 & 16 \\
HOCM & 1 & 2 \\
Takotsubo & 2 & 4 \\
Myocarditis & 3 & 6 \\
Valvular heart disease & 3 & 6 \\
Mitral regurgitation & 1 & 2 \\
Tricuspid regurgitation & 1 & 2 \\
Aortic regurgitation & 1 & 2 \\
Suspected cardiac amyloidosis & 1 & 2 \\
\hline
\end{tabular}

\section{Performance of fully automated algorithm- compared to expert corrected results}

Results of fully automated and expert corrected analyses are summarized in Table 3. The fully automated analysis slightly underestimated the values for left ventricular enddiastolic volume $(p=0.0004)$, stroke volume $(p<0.0001)$ and ejection fraction $(\mathrm{p}<0.0001)$ compared with the expert corrected findings. The end-systolic volume $(p=0.0008)$ was overestimated. Differences in the left ventricular mass between the two analysis were not statistically significant.

The correlation between fully automated results and expert-corrected results was very strong with correlation coefficients of 0.998 (95\% confidence interval 0.995-0.999) for end-diastolic volume, 0.997 (0.995-0.999) for end-systolic volume, $0.899(0.733-0.953)$ for stroke volume, 0.972 (0.923-0.987) for ejection fraction and $0.991(0.985-0.995)$ for myocardial mass (all p $<0.001$, Table 4, Fig. 3). Compared with the expert-corrected results, the fully automated algorithm showed a mean deviation of $-2.1 \%$ for end-diastolic volume, $+3 \%$ for end-systolic volume, $-10 \%$ for stroke volume and $-7.5 \%$ for ejection fraction (Table 4, Fig. 3). Correlations were stronger and limits of agreement narrower for fully automated vs. expert corrected results than between the manual analysis of two radiology trainees (Table 4 and Supplementary Table 1). Similarly, there was a trend for intra-class correlation coefficients to be higher for automated vs. expert corrected results than between the manual analysis of two radiology trainees (Table 4 and Supplementary Table 1), but differences were not statistically significant.

\section{Re-classification rate of LV function}

Compared with the fully automated results, expert corrections resulted in a change in the functional category in 9 of 50 cases (18\%). In 8 of these 9 cases, expert corrections placed the patient in a better functional category, most commonly $(n=4)$ moderate dysfunction (EF 30-39\%) instead of severe dysfunction $(<30 \%)$. A remarkably similar re-classification rate ( 5 of 25 patients, 20\%) was observed between the two manual readers.

\section{Performance in specific sub-groups}

Median end-diastolic volume and ejection fraction were $130.9 \mathrm{ml} / 59.1 \%$ in patients with no pathological findings at cardiac MR, $162.0 \mathrm{ml} / 47.8 \%$ in patients with ischemic heart disease and $244.2 \mathrm{ml} / 28.8 \%$ in patients with cardiomyopathies (Supplementary Table 2). Relative limits of agreement for end-diastolic volume and end-systolic volume were similar across all subgroups. However, for stroke volume and ejection fraction they were wider in ischemic heart disease and cardiomyopathies than in patients without pathological 


\section{Fully automated}
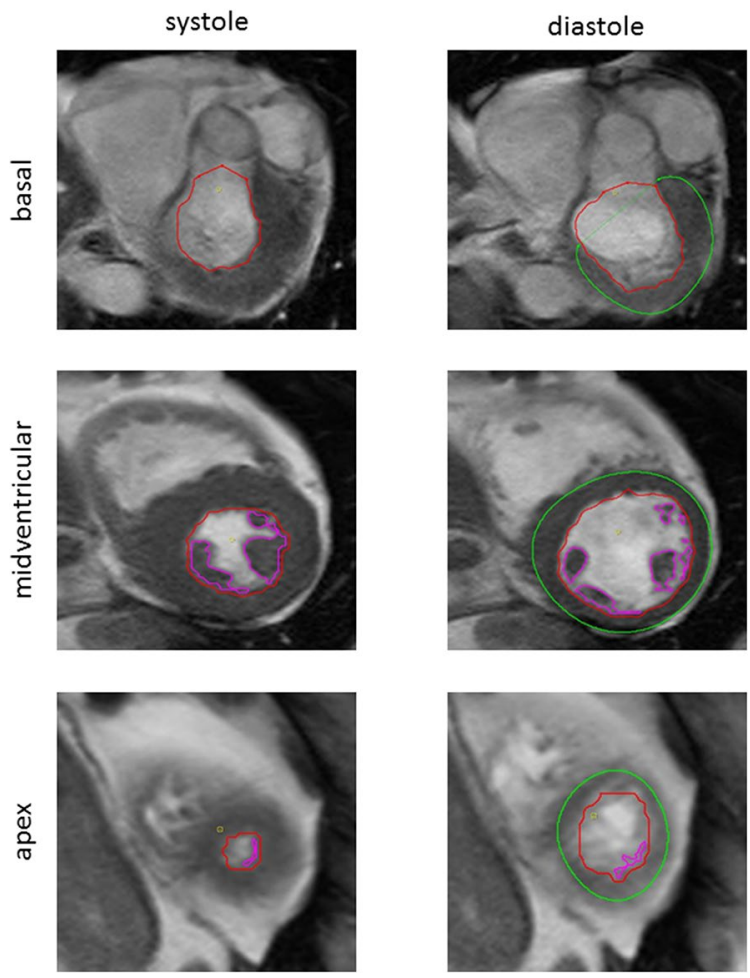

Fig. 1 Representative example of accurate fully automated contouring. Short axis cine MRI sequence of a 77-year old man with no pathological findings. Visualized are the fully automated generated contours of apex, midventricular and basal slices in systole (left) and diastole (right). Contours are shown in red (subendocardial), pink (papillary muscles) and green (subepicardial). No manual corrections were necessary in this case. LV ejection fraction was $65.3 \%$ for both fully automated and expert corrected analysis

findings at cardiac MR (Supplementary Table 3). Intra-class correlation coefficients did not show significant differences between subgroups.

\section{Time required of fully automated, expert corrected and manual volumetric analysis}

The fully automated volumetric analysis required a median of $8.4 \mathrm{~s}$ to complete (interquartile range 8.2-8.6 s). The expertcorrected analysis was completed in a median of $110 \mathrm{~s}$ (IQ range 66-126 s). This includes performing the fully automated analysis, checking correct identification of end-systole and end-diastole, checking correct slice inclusion and accurate contouring and making any necessary corrections. The time required for manual volumetric analysis was approximately $3.5 \mathrm{~min}$ for a cardiovascular imaging fellow (median $210 \mathrm{~s}$, IQ range 183-280 s) and approximately 9 min for a radiology resident (median $525 \mathrm{~s}$, IQ range 461-583 s, p $<0.001$ for all pair-wise comparisons).

\section{Discussion}

In this paper, we investigated the performance of a deep learning-based algorithm for fully automated quantification of LV volumes and function in cardiac MRI. We observed that the fully automated analysis was performed successfully in all patients in less than $10 \mathrm{~s}$. The correlation between fully automated and expert-corrected results was very strongstronger than between the manual analysis of two radiology trainees. Minor corrections were made in most $(80 \%)$ cases. However, even with these corrections the expert corrected automated analysis took less than $2 \mathrm{~min}$ on average-significantly less than the manual analysis, which on average took 3.5 or $9 \mathrm{~min}$, depending on level of experience.

Several previous publications focus on the technical aspects of developing and validating deep learning-based algorithms for fully automated volumetric analysis of cardiac MRI [12, 16-19]. Bai and colleagues describe the development and validation of a fully automated method capable of segmenting the volume of both ventricles and atria based on a multi-layered fully convolutional network [12]. In their study, cardiac MRI images of approximately 5000 subjects from the population-based UK Biobank cohort were manually annotated. Approximately 4000 were used to train the algorithm, 300 served as the validation cohort and 600 datasets were used for testing its performance. In this cohort of predominantly normal cardiac MRIs, they found that the agreement between fully automated computer analysis and human analysis was comparable to the inter-reader agreement between two human readers.

Bernard and colleagues recently provided an in-depth review of deep-learning techniques for automatic cardiac MRI segmentation [11]. They summarize the results obtained by various deep learning-based algorithms on the "Automatic Cardiac Diagnosis Challenge" dataset, a publicly available fully annotated dataset of 150 cardiac MRI examinations. The comparison of multiple algorithms tested on an identical dataset allowed the authors to identify typical challenges for deep learning based cardiac MRI segmentation. In particular, they noted that segmentation results at the base (near the valves) and the apex are most error prone [11]. This is consistent with the result of our study that expert changes to the fully automated segmentation were often related to slice inclusion at the apex or-less commonly - the base of the LV. It is worth mentioning that in our study the correction-rate at the base is lower than in the investigation of Bernard et al. [11].

An important use case for fully automated volumetric analysis are large population-based cohort studies incorporating cardiac MRI examinations. The UK Biobank is on its way to acquire 100,000 cardiac MRI examinations by 2020 [10] and the German National Cohort has performed 


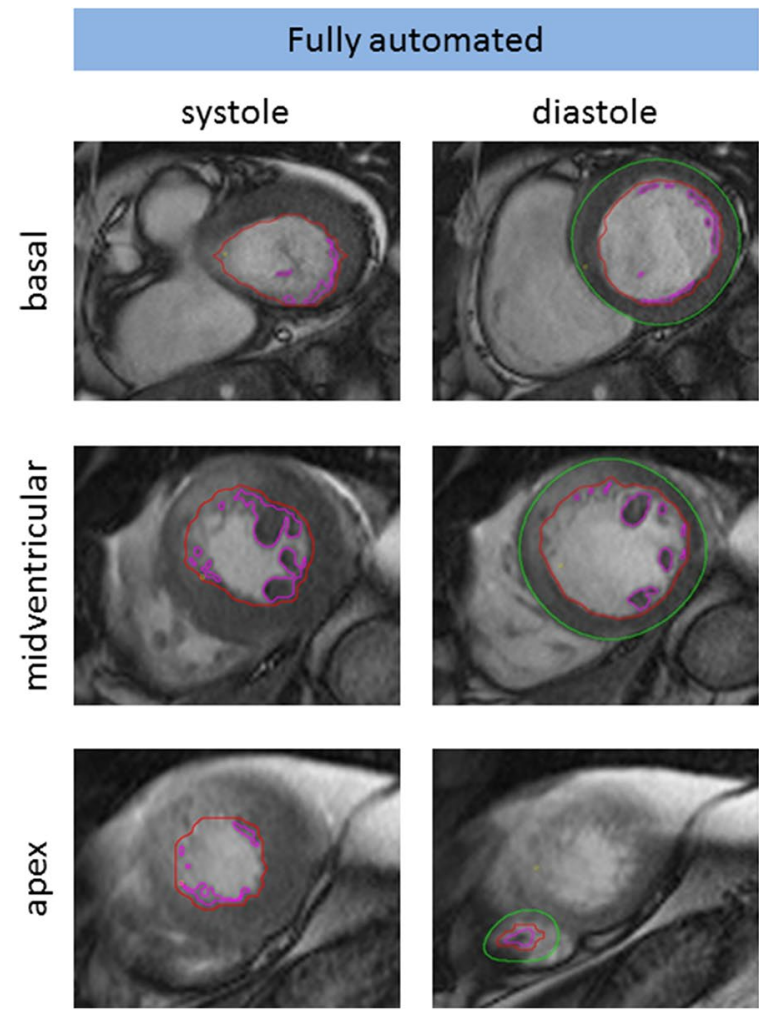

Fig. 2 Representative example of fully automated contouring with corrections. Short axis cine sequence of a 63-year old man with acute myocardial infarction in the anterior wall. Visualized are the fully automated (left two columns) and expert-corrected (right two columns) contours of apex, midventricular and basal slices in systole (left) and diastole (right). Contours are shown in red (subendo-
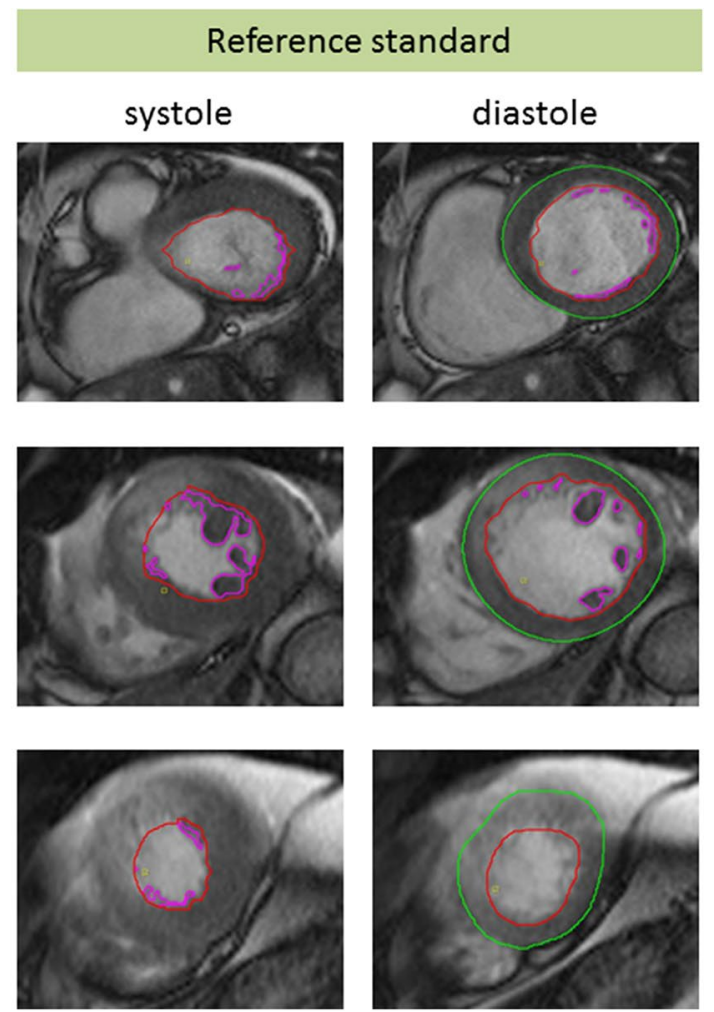

cardial), pink (papillary muscles) and green (subepicardial). In this case, the fully automated contouring made an error in the apical slice by setting contours in the right ventricle instead of the left ventricle. Some minor adjustments of contours were also manually performed. LV ejection fraction was $48.8 \%$ for fully automated and $55.7 \%$ expert corrected analysis
Table 3 Left ventricular parameters in fully automated and expert corrected analysis

\begin{tabular}{|c|c|c|c|c|c|}
\hline & \multicolumn{2}{|c|}{ Fully automated } & \multicolumn{2}{|c|}{ Expert corrected } & \multirow[t]{2}{*}{$\mathrm{P}$ value } \\
\hline & Median & IQR & Median & IQR & \\
\hline \multicolumn{6}{|l|}{ Absolute values } \\
\hline LV EDV (ml) & 160.9 & 124.6-211.8 & 162.4 & $126.7-213.1$ & 0.0004 \\
\hline LV ESV (ml) & 73.1 & $48.5-121.3$ & 66.1 & $46.3-118.9$ & 0.0008 \\
\hline LV SV (ml) & 78.8 & $59.4-95.0$ & 83.7 & $68.7-98.6$ & $<0.0001$ \\
\hline LV EF (\%) & 52.2 & $41.0-62.2$ & 56.0 & $42.6-62.7$ & $<0.0001$ \\
\hline LV mass (g) & 166.5 & 134.4-194.8 & 165.5 & 133.0-195.8 & 0.7677 \\
\hline \multicolumn{6}{|c|}{ Indexed to body surface area } \\
\hline $\operatorname{LV} \operatorname{EDV}\left(\mathrm{ml} / \mathrm{m}^{2}\right)$ & 78.3 & $63.4-98.3$ & 79.2 & $65.6-100.2$ & 0.0005 \\
\hline $\operatorname{LV} \operatorname{ESV}\left(\mathrm{ml} / \mathrm{m}^{2}\right)$ & 35.1 & $25.7-59.5$ & 32.5 & $25.1-59.0$ & 0.0005 \\
\hline $\operatorname{LV~SV~}\left(\mathrm{ml} / \mathrm{m}^{2}\right)$ & 39.8 & $28.4-45.5$ & 41.4 & $34.0-49.9$ & $<0.0001$ \\
\hline LV mass $\left(\mathrm{g} / \mathrm{m}^{2}\right)$ & 81.1 & $64.3-91.9$ & 80.7 & $66.4-89.8$ & 0.7998 \\
\hline
\end{tabular}

$\mathrm{P}$ value is for comparison of fully automated and expert corrected results using Wilcoxon matched pairs test

30,000 whole-body MRI examinations (including cardiac MRI sequences) [20]. It is obvious that such case volumes cannot reasonably be analysed manually but ideally require fully automated approaches. Quality control is key in this effort to ensure the validity of data. Using data from the UK Biobank, important advances have been made in developing a fully automated pipeline for image analysis that includes 
Table 4 Agreement between fully automated and expert corrected quantification

\begin{tabular}{|c|c|c|c|c|c|}
\hline & \multicolumn{2}{|c|}{ Bland-Altmann analysis } & \multicolumn{3}{|l|}{ Correlation analysis } \\
\hline & Mean bias (\%) & Limits of agreement (\%) & $\begin{array}{l}\text { Intra-class correlation } \\
\text { coefficient }\end{array}$ & $95 \%$ confidence interval & $P$ value \\
\hline LV EDV (ml) & -2.1 & $-12.9 /+8.6$ & 0.998 & $0.995-0.999$ & $<0.0001$ \\
\hline LV ESV (ml) & +3 & $-11.7 /+17.9$ & 0.997 & $0.995-0.999$ & $<0.0001$ \\
\hline LV SV (ml) & -10 & $-43.1 /+23.0$ & 0.899 & $0.733-0.953$ & $<0.0001$ \\
\hline LV EF (\%) & -7.5 & $-33.9 /+18.9$ & 0.972 & $0.923-0.987$ & $<0.0001$ \\
\hline LV mass (g) & +0.3 & $-10.5 /+11.0$ & 0.991 & $0.985-0.995$ & $<0.0001$ \\
\hline
\end{tabular}

automated checks for image quality as well as quality and consistency of output $[10,12,14]$.

There is scarce data on the performance and utility of such algorithms in clinical routine. One recent study investigated a similar algorithm by a different vendor in 300 cases randomly selected cases from routine clinical care [13]. Their study found that agreement between fully automated and expert manual segmentation was lower for the right ventricle than for the left ventricle, lower at $3 \mathrm{~T}$, in cases of compromised image quality and in cases of challenging anatomy such as repaired Tetralogy of Fallot [13]. Our study design builds on these results in several aspects: we focused our investigation on the left ventricle and chose to exclude examinations performed at $3 \mathrm{~T}$, examinations with severe artefacts and substantially altered anatomy (severe congenital heart disease, large left ventricular aneurysms).

Our results agree with this previous publication in that both studies indicated that the relative differences are somewhat larger for LV stroke volume and ejection fraction than for end-diastolic volume, end-systolic volume and myocardial mass. This is likely mostly a mathematical effect: Stroke volume and ejection fraction are not measured directly but calculated from end-diastolic and end-systolic volume such that measurement errors add up if errors occur in opposite directions. We furthermore demonstrated that agreement between fully automated and expert-corrected results for stroke volume and ejection fraction was lower in ischemic heart disease and cardiomyopathies than in patients without pathological findings at cardiac MR. In part, this may be attributed to the lower ejection fraction in these patients, which means that the same absolute deviations amount to wider relative limits of agreement. In part, however, this may also be an expression of how the algorithm was developed, namely initially trained on a large volume of - mostly normal-cardiac MRIs from the population-based UK Biobank cohort, although the algorithm was later also trained on a large volume of pathological cases.

To establish a precise reference standard for our analysis, meticulous corrections to the fully automated contours were made by an expert in our study in as many as $80 \%$ of cases. However, many of these corrections were minor and may not be clinically meaningful. To address this issue, we performed a secondary analysis of the re-classification rate based on pre-specified categories of LV function. We found that expert corrections made a clinically meaningful difference in $18 \%$ of cases, very similar to the re-classification rate between the two manual readers ( $20 \%$ in our study). This suggests that only a minority of cases may require corrections in clinical routine. However, we do not recommend using the algorithm without supervision. Rather, the results of fully automated volumetric analysis should be checked and corrected, if necessary, by a human reader. As with other applications of artificial intelligence, the role of humans imaging experts shifts from performing mechanical tasks to critically reflecting the results of algorithms and developing a professional relationship between physicians, patients and machine-generated data [21].

Several limitations of our study are worth noting. This was a retrospective analysis describing our initial experience in a limited number of cases. In particular, the number of patients in each subgroup was relatively small, such that the results of the subgroup analysis should be considered hypothesis-generating. An obvious limitation of our investigation is that we examined the performance of a specific deep learning-based fully automated algorithm. The results cannot be directly transferred to the algorithms of other vendors or even later version of the same algorithm. However, we believe that our results may provide some insights into strengths and typical limitations of deep learning-based approaches to fully automated volumetric CMR analysis that may benefit the further development of the technique.

In conclusion, deep learning-based fully automated analysis of left ventricular volumes and function is feasible, extremely fast and shows respectable performance without any manual corrections. Even with manual correctionswhich are required for precise results in most patientsthis approach remains time-efficient compared to manual analysis. 
Fig. 3 Agreement between fully automated and expert corrected left ventricular volumes and function. Scatter plots (left column) visualize correlation between fully automated (Y-axis) and expert corrected results (X-axis). Bland-Altman plots (right column) show relative difference (in \%) between fully automated and expert corrected results (Y-axis) as a function of absolute values (average of both methods shown on the $\mathrm{X}$-axis). The blue dotted lines indicate limits of agreement (95\% of all values). The red dotted line indicates the bias (mean difference). $E D V$ End-diastolic volume, $E S V$ end-systolic volume, $S V$ stroke volume, $E F$ ejection fraction
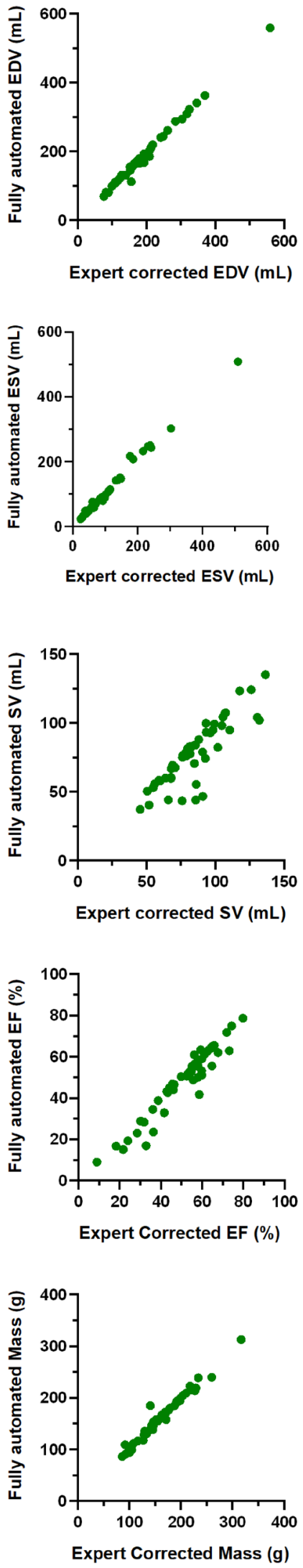
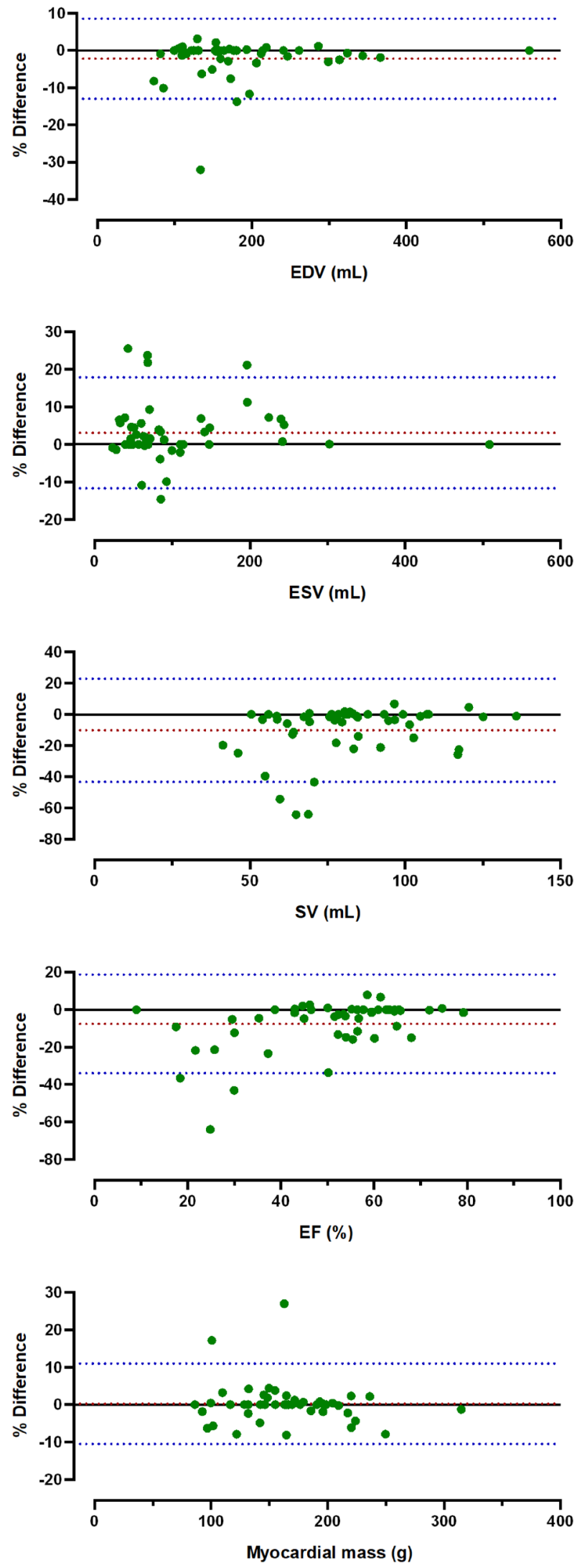
Acknowledgements Open Access funding provided by Projekt DEAL.

\section{Compliance with ethical standards}

Conflict of interest The authors declare that they have no conflics of interest.

Open Access This article is licensed under a Creative Commons Attribution 4.0 International License, which permits use, sharing, adaptation, distribution and reproduction in any medium or format, as long as you give appropriate credit to the original author(s) and the source, provide a link to the Creative Commons licence, and indicate if changes were made. The images or other third party material in this article are included in the article's Creative Commons licence, unless indicated otherwise in a credit line to the material. If material is not included in the article's Creative Commons licence and your intended use is not permitted by statutory regulation or exceeds the permitted use, you will need to obtain permission directly from the copyright holder. To view a copy of this licence, visit http://creativecommons.org/licenses/by/4.0/.

\section{References}

1. Perelshtein Brezinov O, Klempfner R, Zekry SB et al (2017) Prognostic value of ejection fraction in patients admitted with acute coronary syndrome: a real world study. Medicine (Baltimore). https://doi.org/10.1097/MD.0000000000006226

2. Burns RJ, Gibbons RJ, Yi Q et al (2002) The relationships of left ventricular ejection fraction, end-systolic volume index and infarct size to six-month mortality after hospital discharge following myocardial infarction treated by thrombolysis. J Am Coll Cardiol 39(1):30-36. https://doi.org/10.1016/s0735-1097(01)01711-9

3. Anzini M, Merlo M, Sabbadini G et al (2013) Long-term evolution and prognostic stratification of biopsy-proven active myocarditis. Circulation 128(22):2384-2394. https://doi.org/10.1161/CIRCU LATIONAHA.113.003092

4. Solomon SD, Anavekar N, Skali H et al (2005) Influence of ejection fraction on cardiovascular outcomes in a broad spectrum of heart failure patients. Circulation 112(24):3738-3744. https://doi. org/10.1161/CIRCULATIONAHA.105.561423

5. Ponikowski P, Voors AA, Anker SD et al (2016) 2016 ESC Guidelines for the diagnosis and treatment of acute and chronic heart failure: the Task Force for the diagnosis and treatment of acute and chronic heart failure of the European Society of Cardiology (ESC) developed with the special contribution of the Heart Failure Association (HFA) of the ESC. Eur Heart J 37(27):2129-2200. https://doi.org/10.1093/eurheartj/ehw128

6. Russo AM, Stainback RF, Bailey SR et al (2013) ACCF/HRS/ AHA/ASE/HFSA/SCAI/SCCT/SCMR 2013 appropriate use criteria for implantable cardioverter-defibrillators and cardiac resynchronization therapy: a report of the American College of Cardiology Foundation appropriate use criteria task force, Heart Rhythm Society, American Heart Association, American Society of Echocardiography, Heart Failure Society of America, Society for Cardiovascular Angiography and Interventions, Society of Cardiovascular Computed Tomography, and Society for Cardiovascular Magnetic Resonance. J Am Coll Cardiol 61(12):1318 1368. https://doi.org/10.1016/j.jacc.2012.12.017

7. Doherty JU, Kort S, Mehran R et al (2019) ACC/AATS/AHA/ ASE/ASNC/HRS/SCAI/SCCT/SCMR/STS 2019 appropriate use criteria for multimodality imaging in the assessment of cardiac structure and function in nonvalvular heart disease: a report of the American College of Cardiology Appropriate Use Criteria Task Force, American Association for Thoracic Surgery, American
Heart Association, American Society of Echocardiography, American Society of Nuclear Cardiology, Heart Rhythm Society, Society for Cardiovascular Angiography and Interventions, Society of Cardiovascular Computed Tomography, Society for Cardiovascular Magnetic Resonance, and the Society of Thoracic Surgeons. J Am Coll Cardiol 73(4):488-516. https://doi. org/10.1016/j.jacc.2018.10.038

8. Litjens G, Ciompi F, Wolterink JM et al (2019) State-of-the-art deep learning in cardiovascular image analysis. JACC Cardiovasc Imaging 12(8 Pt 1):1549-1565

9. Queirós S, Barbosa D, Engvall J et al (2016) Multi-centre validation of an automatic algorithm for fast 4D myocardial segmentation in cine CMR datasets. Eur Heart J Cardiovasc Imaging 17(10):1118-1127. https://doi.org/10.1093/ehjci/jev247

10. Suinesiaputra A, Sanghvi MM, Aung N et al (2018) Fully-automated left ventricular mass and volume MRI analysis in the UK Biobank population cohort: evaluation of initial results. Int J Cardiovasc Imaging 34(2):281-291. https://doi.org/10.1007/s 1055 4-017-1225-9

11. Bernard O, Lalande A, Zotti C et al (2018) Deep learning techniques for automatic MRI cardiac multi-structures segmentation and diagnosis: is the problem solved? IEEE Trans Med Imaging 37(11):2514-2525. https://doi.org/10.1109/TMI.2018.2837502

12. Bai W, Sinclair M, Tarroni G et al (2018) Automated cardiovascular magnetic resonance image analysis with fully convolutional networks. J Cardiovasc Magn Reson 20(1):65. https://doi. org/10.1186/s12968-018-0471-x

13. Backhaus SJ, Staab W, Steinmetz M et al (2019) Fully automated quantification of biventricular volumes and function in cardiovascular magnetic resonance: applicability to clinical routine settings. J Cardiovasc Magn Reson 21(1):24. https://doi.org/10.1186/s1296 8-019-0532-9

14. Ruijsink B, Puyol-Antón E, Oksuz I et al (2019) Fully automated, quality-controlled cardiac analysis from CMR: validation and large-scale application to characterize cardiac function. JACC Cardiovasc Imaging. 20:19. https://doi.org/10.1016/j. jcmg.2019.05.030

15. American College of Cardiology. https://www.acc.org/tools-andpractice-support/clinical-toolkits/heart-failure-practice-solutions/ left-ventricular-ejection-fraction-lvef-assessment-outpatient-setti ng. Accessed 19 Dec 2019

16. Avendi MR, Kheradvar A, Jafarkhani H (2016) A combined deeplearning and deformable-model approach to fully automatic segmentation of the left ventricle in cardiac MRI. Med Image Anal 30:108-119. https://doi.org/10.1016/j.media.2016.01.005

17. Ma Z, Wu X, Wang X et al (2019) An iterative multi-path fully convolutional neural network for automatic cardiac segmentation in cine MR images. Med Phys 37:2514. https://doi.org/10.1002/mp.13859

18. Dangi S, Linte CA, Yaniv Z (2019) A distance map regularized CNN for cardiac cine MR image segmentation. Med Phys. https ://doi.org/10.1002/mp.13853

19. Vigneault DM, Xie W, Ho CY et al (2018) $\Omega$-Net (Omega-Net): fully automatic, multi-view cardiac MR detection, orientation, and segmentation with deep neural networks. Med Image Anal 48:95-106. https://doi.org/10.1016/j.media.2018.05.008

20. Bamberg F, Kauczor H-U, Weckbach S et al (2015) Whole-body MR imaging in the German National Cohort: rationale, design, and technical background. Radiology 277(1):206-220. https://doi. org/10.1148/radiol.2015142272

21. de Souza M, Filho E, de Amorim Fernandes F, de Abreu L, Soares C et al (2019) Inteligência Artificial em Cardiologia: Conceitos, Ferramentas e Desafios - "Quem Corre é o Cavalo, Você Precisa ser o Jóquei”. ABC Cardiol. https://doi.org/10.36660/abc.20180431

Publisher's Note Springer Nature remains neutral with regard to jurisdictional claims in published maps and institutional affiliations. 